\title{
Some Properties of Fuzzy Sets on Finite Type of Kac-Moody Algebra $\mathrm{G}_{2}$
}

\author{
A. Uma Maheswari \\ Associate Professor \\ Quaid-E-Millath Government College for Women \\ (Autonomous), \\ Chennai -600002
}

\author{
S.Krishnaveni \\ Associate Professor \\ M.O.P. Vaishnav College for Women \\ (Autonomous), \\ Chennai - 600034
}

\begin{abstract}
The Kac-Moody algebras has been attracting the attention of a lot of Mathematicians because of its various connections and applications to different branches of Mathematics and Mathematical Physics since the introduction of the subject in 1968, developed simultaneously and independently by Kac and Moody. On the other hand, Fuzzy sets first originated in a seminar paper by Lotfi A.Zadeh in 1965. The theory on fuzzy sets has been applied not only to all branches of Mathematics but also acts as a tool for solving challenging problems in science \& technology and social problems.
\end{abstract}

Fuzzy approach on Kac-Moody algebras was initiated by A.Uma Maheswari [4] in which fuzzy sets were defined on the root system of Kac-Moody algebras. Some fuzzy properties were studied for the finite type of Kac-Moody algebras $\mathrm{A}_{l}, \mathrm{~B}_{l}, \mathrm{C}_{l}, \mathrm{D}_{l}, \mathrm{E}_{6}, \mathrm{E}_{7}, \mathrm{E}_{8}$, in [4], [7] \& [9], for the affine type of Kac-Moody algebras in [5] \& [6] and hyperbolic type $\mathrm{HG}_{2}$ in [8]. In this paper another fuzzy approach is attempted on the root system of finite type of Kac-Moody algebra $\mathrm{G}_{2}$.

Fuzzy sets are defined on the cartesian product of the root basis of $\mathrm{G}_{2}$ using an invariant, non degenerate, symmetric bilinear form. Some of the basic properties like support, core, normality, height, cardinality, relative cardinality, complement and convexity are studied; $\alpha$-level sets and strong $\alpha$-level sets are computed. $\alpha$-cut decomposition, hamming distance and euclidean distance for the fuzzy set associated with $\mathrm{G}_{2}$ of finite type of Kac-Moody algebra are also computed.

\section{Keywords}

Generlized Cartan Matrix, Kac-Moody algebra, root basis, non- degenerate form, fuzzy set, core, normal, height, convexity, $\alpha$-level set, strong $\alpha$-level set, $\alpha$ - cut decomposition.

\section{INTRODUCTION}

\subsection{Basic definitions of Fuzzy Sets}

Definition 1 [11]: A classical (crisp) set is normally defined as a collection of elements or objects $\mathrm{x} \in \mathrm{X}$ that can be finite, countable or overcountable

Definition 2 [11]: If $X$ is a collection of objects denoted generically by $x$, then a fuzzy set

$\widetilde{\mathrm{A}}=\left\{\left(\mathrm{x}, \mu_{\tilde{\mathrm{A}}}(\mathrm{x})\right) / \mathrm{x} \in \mathrm{X}\right\} \cdot \mu_{\tilde{\mathrm{A}}}(\mathrm{x})$ is called the "membership function" or "grade of membership" of $\mathrm{x}$ in $\tilde{\mathrm{A}}$ that maps $\mathrm{X}$ to the membership space $\mathrm{M}$.
Definition 3 [11]: The support of a fuzzy set $\tilde{A}, S(\tilde{A})$ is the crisp set of all $\mathrm{x} \in \mathrm{X}$ such that $\mu_{\tilde{A}}(x)>0$.

Definition 4 [11]: The (crisp) set of elements that belong to the fuzzy set $\tilde{\mathrm{A}}$, at least to the degree $\alpha$ is called the $\alpha$ level set

$A_{\alpha}=\left\{x \in X / \mu_{\tilde{A}}(x) \geq 0\right\}$,

$\mathrm{A}_{\alpha}^{\prime}=\left\{\mathrm{x} \in \mathrm{X} / \mu_{\tilde{\mathrm{A}}}(\mathrm{x})>0\right\}$, is called strong $\alpha$ - level set or strong $\alpha$-cut.

Definition 5 [11]: Let $\tilde{\mathrm{A}}$ be a fuzzy set on $\mathrm{X}$. Then the set $\left\{\mathrm{x} \in \mathrm{X} / \mu_{\tilde{\mathrm{A}}}(\mathrm{x})=1\right\}$ is called the core of the fuzzy set $\tilde{\mathrm{A}}$. This set is denoted by core $(\tilde{\mathrm{A}})$. A fuzzy set $\tilde{\mathrm{A}}$ is said to be normal if $\sup _{\mathrm{x}} \mu_{\tilde{\mathrm{A}}}(\mathrm{x})=1$.

Definition 6 [11]: The height of a fuzzy set is the largest membership grade attained by any element in that set.

Definition 7 [11]: A fuzzy set $\tilde{A}$ is convex if $\mu_{\tilde{A}}\left[\lambda x_{1}+(1-\lambda) x_{2}\right] \geq \min \left[\mu_{\tilde{A}}\left(x_{1}\right), \mu_{\tilde{A}}\left(x_{2}\right)\right] \forall \mathrm{x}_{1}, x_{2} \in \mathrm{X}$ and $\lambda \in[0,1]$. Alternatively, a fuzzy set is convex if all $\alpha$ - level sets are convex.

Definition 8 [1]: Let $A$ be a fuzzy set on $U$ and $\alpha$ be a number such that $0<\alpha \leq 1$. Then by $\alpha \mathrm{A}$ we mean a fuzzy set on $\mathrm{U}$, denoted by $\alpha \mathrm{A}$ which is such that $(\alpha \mathrm{A})(\mathrm{x})=\alpha$ $A(x)$ for every $x$ in $U$. This procedure of associating another fuzzy set with the given fuzzy set A is termed as restricted scalar multiplication.

Definition 9 [1]: Any fuzzy set A on U can be decomposed as $\mathrm{A}=\sup \left\{\alpha \mathrm{A}_{\alpha} / 0<\alpha \leq 1\right\}$. we also write $\mathrm{A}=\sum \alpha \mathrm{A}_{\alpha}($ or $) \mathrm{A}=\cup \alpha \mathrm{A}_{\alpha}$

Definition 10 [1]: Complement of a fuzzy set A, denoted by $\mathrm{A}^{\prime}$, is defined as the fuzzy set on $\mathrm{U}$, for which $\left(\mathrm{A}^{\prime}\right)(\mathrm{x})=1-$ $\mathrm{A}(\mathrm{x})$ for every $\mathrm{x}$ in $\mathrm{U}$.

Definition 11 [1]: Let A be a fuzzy set on U. Then by the scalar cardinality of A we mean the number $\sum \mathrm{A}(\mathrm{x})$ where the summation is over all the elements of $\mathrm{U}$. 
This number is denoted by $|A|$ or SC(A).

Definition 12 [1]: Let A be a fuzzy set on $U$, we associate $\mathrm{L}(\mathrm{A})$, a crisp subset on $\mathrm{I}=(0,1]$, called its level set, $\mathrm{L}(\mathrm{A})$ is defined as follows:

$\mathrm{L}(\mathrm{A})=\{\alpha \in \mathrm{I} \mid \mathrm{A}(\mathrm{x})=\alpha$ for some $\mathrm{x} \in \mathrm{U}\}$

Definition 13 [11]: For a finite fuzzy set $\tilde{A}$, the cardinality $|\tilde{A}|$ is defined as $|\tilde{A}|=\sum_{x \in X} \mu_{\tilde{A}}(x)$

$\|\tilde{A}\|=\frac{|\tilde{A}|}{|X|}$ is called the relative cardinality of $\tilde{A}$.

Definition 14 [1]: Let A be a non-empty fuzzy set on $U$ and $\operatorname{supp}(\mathrm{A})$ be finite. Its fuzzy cardinality, denoted by $\mathrm{FC}(\mathrm{A})$, is defined as the fuzzy set on $\mathrm{N}$ (the set of all the natural numbers) given by $\sum \alpha / \mathrm{SC}\left(\mathrm{A}_{\alpha}\right)$, i.e. $\mathrm{FC}(\mathrm{A})=\sum \alpha / \mathrm{n}_{\alpha}$ where $\mathrm{n}_{\alpha}=\mathrm{SC}\left(\mathrm{A}_{\alpha}\right)$ and $\sum$ runs over all $\alpha$ in $\mathrm{L}(\mathrm{A})$ [here $\mathrm{N}=\{1,2,3, \ldots\}$.

Definition 15 [1]: For a fuzzy set $A$ on $\mathrm{U}$, and a positive real number $\alpha$, we define the $\alpha$-th power of A (denoted by $\left.\mathrm{A}^{\alpha}\right), \mathrm{A}^{\alpha}(\mathrm{x})=[\mathrm{A}(\mathrm{x})]^{\alpha}$ for all $\mathrm{x}$ in $\mathrm{U}$.

Definition 16 [1]: Dilation of A is denoted by Dil(A) and is given $\operatorname{Dil}(\mathrm{A})=[\mathrm{A}(\mathrm{x})]^{0.5}$ for all $\mathrm{x}$ in $\mathrm{U}$, that is, $\operatorname{Dil}(\mathrm{A})=\mathrm{A}^{0.5}$

Definition 17 [1]: The Concentration is denoted by con(A) and is given by con $(\mathrm{A})(\mathrm{x})=[\mathrm{A}(\mathrm{x})]^{2}$ for $\mathrm{x}$ in $\mathrm{U}$.

Definition 18 [1]: The Contrast Intensification of a fuzzy set A , denoted by $\operatorname{Int}(\mathrm{A})$, is defined as :

$\operatorname{Int}(\mathrm{A})(\mathrm{x})=2[\mathrm{~A}(\mathrm{x})]^{2}$ for $0 \leq \mathrm{A}(\mathrm{x}) \leq 0.5$

$$
=1-2[1-\mathrm{A}(\mathrm{x})]^{2} \text { for } 0.5 \leq \mathrm{A}(\mathrm{x}) \leq 1
$$

Definition 19 [1]: Index of Fuzziness indicates a method of measuring the fuzziness associated with a fuzzy set. This index is given by the following functions:

$\operatorname{Hamm}(\mathrm{A})=\sum\left|\mathrm{A}(\mathrm{x})-\mathrm{A}^{\prime}(\mathrm{x})\right|$

$\operatorname{Euc}(\mathrm{A})=\left[\sum\left(\mathrm{A}(\mathrm{x})-\mathrm{A}^{\prime}(\mathrm{x})\right)^{2}\right]^{1 / 2}$

where the summation signs are taken over all elements of $\operatorname{supp}(\mathrm{A})$ [assuming $\operatorname{supp}(\mathrm{A})$ to be finite]. Hamm is called hamming distance and Euc is called euclidean distance. Note that $\operatorname{Hamm}(\mathrm{A})=$ the cardinality of $\operatorname{supp}(\mathrm{A})$, if $\mathrm{A}$ is a crisp set; and $\operatorname{Hamm}(\mathrm{A})=0$ if $\mathrm{A}(\mathrm{x})=0.5$ for all $\mathrm{x}$ in $\mathrm{U}$.

Thus , $0 \leq \operatorname{Hamm}(\mathrm{A}) \leq \mathrm{n}$, where $\mathrm{n}$ is the cardinality of $\operatorname{supp}(\mathrm{A})$.

Another approach is also available which is based on the $\alpha$ cut with $\alpha=0.5$. The index is based on this idea is given by

$\mathrm{f}_{\mathrm{H}}(\mathrm{A})=$

$\sum\left|\mathrm{A}(\mathrm{x})-\mathrm{A}_{0.5}(\mathrm{x})\right|, \mathrm{f}_{\mathrm{E}}(\mathrm{A})=\left[\sum\left(\mathrm{A}(\mathrm{x})-\mathrm{A}_{0.5}(\mathrm{x})\right)^{2}\right]^{1 / 2}$

Where the summation is over all the elements in $\operatorname{supp}(\mathrm{A})$ is finite. These can be normalized in case $\operatorname{supp}(\mathrm{A})$ is finite, so that the normalized values lie between 0 and 1 . We have the following:

$\mathrm{f}_{\mathrm{H}}{ }^{*}(\mathrm{~A})=\frac{\left(2 \mathrm{f}_{\mathrm{H}}(\mathrm{A})\right)}{\#(\operatorname{supp}(\mathrm{A}))}, \mathrm{f}_{\mathrm{E}}^{*}(\mathrm{~A})=\frac{\left(2 \mathrm{f}_{\mathrm{E}}(\mathrm{A})\right)}{\left[\#(\operatorname{supp}(\mathrm{A})]^{0.5}\right.}$

\subsection{Basic Definitions on Kac-Moody Algebras}

Definition 20 [3]: An integer matrix $A=\left(a_{i, j}\right)_{i, j=1}^{n}$ is a Generalized Cartan Matrix (abbreviated as GCM) if it satisfies the following conditions:

$\mathrm{a}_{\mathrm{ii}}=2 \quad \forall \mathrm{i}=1,2, \ldots, \mathrm{n}$

$\mathrm{a}_{\mathrm{ij}}=0 \Leftrightarrow \mathrm{a}_{\mathrm{ji}}=0 \forall \mathrm{i}, \mathrm{j}=1,2, \ldots, \mathrm{n}$

$\mathrm{a}_{\mathrm{ij}} \leq 0 \forall \mathrm{i}, \mathrm{j}=1,2, \ldots, \mathrm{n}$.

Let us denote the index set of $\mathrm{A}$ by $\mathrm{N}=\{1, \ldots, \mathrm{n}\}$. A GCM $\mathrm{A}$ is said to decomposable if there exist two non-empty subsets I, $\mathrm{J} \subset \mathrm{N}$ such that $\mathrm{I} \cup \mathrm{J}=\mathrm{N}$ and $\mathrm{a}_{\mathrm{ij}}=\mathrm{a}_{\mathrm{ji}}=0 \forall \mathrm{i} \in \mathrm{I}$ and $\mathrm{j} \in \mathrm{J}$. If $\mathrm{A}$ is not decomposable, it is said to be indecomposable.

Definition 21 [2]: A GCM A is called symmetrizable if DA is symmetric for some diagonal matrix $\mathrm{D}=\operatorname{diag}\left(\mathrm{q}_{1}, \ldots, \mathrm{q}_{\mathrm{n}}\right)$, with $\mathrm{q}_{\mathrm{i}}>0$ and $\mathrm{q}_{\mathrm{i}}$ 's are rational numbers.

Definition 22 [2]: A realization of a matrix $\mathrm{A}=\left(a_{i j}\right)_{\mathrm{i}, \mathrm{j}=1}^{\mathrm{n}}$ is a triple $\left(\mathrm{H}, \Pi, \Pi^{\mathrm{v}}\right)$ where $l$ is the rank of $\mathrm{A}, \mathrm{H}$ is a $2 \mathrm{n}-l$ dimensional complex vector space, $\Pi=\left\{\alpha_{1}, \ldots, \alpha_{n}\right\}$ and $\Pi^{\mathrm{v}}=\left\{\alpha_{1}^{\mathrm{v}}, \ldots, \alpha_{\mathrm{n}}^{\mathrm{v}}\right\}$ are linearly independent subsets of $\mathrm{H}^{*}$ and $H$ respectively, satisfying $\alpha_{j}\left(\alpha_{i}^{v}\right)=a_{i j}$ for $i, j=$ $1, \ldots$, n. $\Pi$ is called the root basis. Elements of $\Pi$ are called simple roots. The root lattice generated by $\Pi$ is $\mathrm{Q}=\sum_{\mathrm{i}=1}^{\mathrm{n}} \mathrm{Z} \alpha_{\mathrm{i}}$

Definition 23 [2]: The Kac-Moody algebra $g(\mathrm{~A})$ associated with a GCM $\mathrm{A}=\left(a_{i j}\right)_{\mathrm{i}, \mathrm{j}=1}^{\mathrm{n}}$ is the Lie algebra generated by the elements $e_{i}, f_{i}, i=1,2, \ldots n$ and $H$ with the following defining relations :

$\left[h, h^{\prime}\right]=0, \quad h, h^{\prime} \in H$

$\left[e_{i}, f_{j}\right]=\delta_{i j} \alpha_{i}^{v}$

$\left[h, e_{j}\right]=\alpha_{j}(h) e_{j}$

$\left[h, f_{j}\right]=-\alpha_{j}(h) f_{j}, i, j \in N$

$\left(a d e_{i}\right)^{1-a_{i j}} e_{j}=0$

$\left(a d f_{i}\right)^{1-a_{i j}} f_{j}=0, \forall \mathbf{i} \neq \mathbf{j}, \mathbf{i}, \mathbf{j} \in \mathrm{N}$

The Kac-Moody algebra $\mathrm{g}(\mathrm{A})$ has the root space decomposition $\mathrm{g}(\mathrm{A})=\underset{\alpha \in \mathrm{Q}}{\oplus} \mathrm{g}_{\alpha}(\mathrm{A})$ where

$\mathrm{g}_{\alpha}(\mathrm{A})=\{\mathrm{x} \in \mathrm{g}(\mathrm{A}) /[\mathrm{h}, \mathrm{x}]=\alpha(\mathrm{h}) \mathrm{x}$, for all $\mathrm{h} \in \mathrm{H}\}$. An element $\alpha, \alpha \neq 0$ in $\mathrm{Q}$ is called a root if $\mathrm{g}_{\alpha} \neq 0$. Let $\mathrm{Q}=\sum_{\mathrm{i}=1}^{\mathrm{n}} \mathrm{Z}_{+} \alpha_{\mathrm{i}}$. Q has a partial ordering " $\leq$ ” defined by $\alpha \leq \beta$ if $\beta-\alpha \in Q+$, where $\alpha, \beta \in Q$

Definition 24 [2]: For any $\alpha \in \mathrm{Q}$ and $\alpha=\sum_{\mathrm{i}=1}^{\mathrm{n}} \mathrm{k}_{\mathrm{i}} \alpha_{\mathrm{i}}$, define support of $\alpha$, written as supp $\alpha$, by $\operatorname{supp} \alpha=\left\{i \in N / k_{i} \neq 0\right\}$. Let $\Delta(=\Delta(A))$ denote the set of all roots of $\mathrm{g}(\mathrm{A})$ and $\Delta_{+}$the set of all positive roots of $\mathrm{g}(\mathrm{A})$. We have $\Delta_{-}=-\Delta_{+}$and $\Delta=\Delta_{+} \cup \Delta_{-}$. 
Proposition 25 [2]: A GCM $A=\left(a_{i, j}\right)_{i, j=1}^{n}$ is symmetrizable if and only if there exists an invariant, bilinear, symmetric, non-degenerate form on $\mathrm{g}(\mathrm{A})$.

Definition 26 [2]: To every GCM A is associated a Dynkin diagram $\mathrm{S}(\mathrm{A})$ defined as follows: $\mathrm{S}(\mathrm{A})$ has $\mathrm{n}$ vertices and vertices $i$ and $j$ are connected by $\max \left\{\left|a_{i j}\right|,\left|a_{j i}\right|\right\}$ number of lines if $a_{i j} \cdot a_{j i} \leq 4$ and there is an arrow pointing towards $i$ if $\left|a_{i j}\right|>1$. If $a_{i j} \cdot a_{j i}>4, i$ and $j$ are connected by a bold faced edge, equipped with the ordered pair $\left(\left|\mathrm{a}_{\mathrm{ij}}\right|,\left|\mathrm{a}_{\mathrm{ji}}\right|\right)$ of integers

Theorem 27 [2]: Let A be a real $\mathrm{n}$ x n matrix satisfying ( $\mathrm{m} 1)$, (m2) and (m3).

(m1) A is indecomposable;

(m2) $\mathrm{a}_{\mathrm{ij}} \leq 0$ for $\mathrm{i} \neq \mathrm{j}$;

(m3) $\quad a_{\mathrm{ij}}=0$ implies $\mathrm{a}_{\mathrm{ji}}=0$

Then one and only one of the following three possibilities holds for both $\mathrm{A}$ and ${ }^{\mathrm{t}} \mathrm{A}$ :

(i) $\operatorname{det} \mathrm{A} \neq 0$; there exists $\mathrm{u}>0$ such that $\mathrm{A} \mathrm{u}>0$; $\mathrm{Av} \geq 0$ implies $\mathrm{v}>0$ or $\mathrm{v}=0$;

(ii) $\operatorname{co} \operatorname{rank} \mathrm{A}=1$; there exists $\mathrm{u}>0$ such that $\mathrm{Au}=0 ; \mathrm{Av} \geq$ 0 implies $\mathrm{Av}=0$;

(iii) there exists $\mathrm{u}>0$ such that $\mathrm{Au}<0 ; \mathrm{Av} \geq 0, \mathrm{v} \geq 0$ imply $\mathrm{v}=0$.

Then A is of finite, affine or indefinite type iff (i), (ii) or (iii) (respectively) is satisfied.

Definition 28 [2]: A Kac- Moody algebra $g(A)$ is said to be of finite, affine or indefinite type if the associated GCM A is of finite, affine or indefinite type respectively.

Definition 29 [10]: A symmetric bilinear C- valued form $\langle.$, . $\rangle$ on a complex Lie algebra $\mathrm{g}(\mathrm{A})$ is said to be invariant if $([x, y], z)=(x,[y, z])$ for all $x, y, z \in g(A)$.

Theorem 30 [10]: For any complex $\mathrm{n} \times \mathrm{n}$ matrix $\mathrm{A}$ the following statements are equivalent:

(a). There exists an invariant, non-degenerate, symmetric,

bilinear $\mathrm{C}$ valued form $<\alpha_{i}, \alpha_{j}>$ on $g(\mathrm{~A})$.

(b). A is s ymmetrizable. Moreover, if these conditions are satisfied, then

(c) $<., .>\mid g_{\alpha} \times g_{\beta}$ is

$=\left\{\begin{array}{l}0 \text { if } \alpha, \beta \in \Delta \cup\{0\} \text { and } \alpha+\beta \neq 0, \\ \text { non degeneratdy paired, if } \alpha, \beta \in \Delta \cup\{0\} \text { and } \alpha+\beta=0\end{array}\right.$

In particular, the restriction of $<.,$.$\rangle to \mathrm{H}$ is also nondegenerate.

Note 31 [4]: Note that for the finite type of Kac-Moody algebra the rank of the GCM A $=$ n.ie $l=\mathrm{n}$.

In paper [4], the new concept of fuzzy sets on the root systems of Kac- Moody algebras was introduced. The fuzzy set on $X=\pi \times \pi$, where $\pi=\left\{\alpha_{1}, \alpha_{2}, \ldots, \alpha_{n}\right\}$, is defined as follows:

$$
\mu_{\tilde{\mathrm{A}}}\left(\alpha_{\mathrm{i}}, \alpha_{\mathrm{j}}\right)=\frac{\left|<\alpha_{\mathrm{i}}, \alpha_{\mathrm{j}}>\right|}{\max \left|<\alpha_{\mathrm{p}}, \alpha_{\mathrm{q}}>\right| \mathrm{p}, \mathrm{q}=1,2, \ldots, \mathrm{n}}
$$

where $<.$, . $>$ denotes bilinear, invariant, non-degenerate symmetric form on $\mathrm{X}$ defined by $<\alpha_{\mathrm{i}}, \alpha_{\mathrm{j}}>=$ bij, where $\mathrm{B}=(\mathrm{bij})$ and $\mathrm{A}=\mathrm{DB}$. Then $\tilde{\mathrm{A}}=\left(\left(\alpha_{\mathrm{i}}, \alpha_{\mathrm{j}}\right), \mu_{\tilde{\mathrm{A}}}\left(\alpha_{\mathrm{i}}, \alpha_{\mathrm{j}}\right)\right)$ forms a fuzzy set on $\pi \times \pi$.

\section{SOME FUZZY PROPERTIES ON THE ROOT SYSTEMS OF FINITE KAC- MOODY ALGEBRA $\mathbf{G}_{\mathbf{2}}$}

Now, for the finite type of Kac-Moody algebra $\mathrm{G}_{2}$, whose Dynkin Diagram is

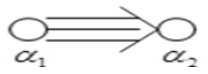

and the associated GCM with $\mathrm{G}_{2}$ is $\mathrm{A}=\left(\begin{array}{cc}2 & -1 \\ -3 & 2\end{array}\right)$ and $\mathrm{A}=$

$\mathrm{D} B$ where $\mathrm{D}=\left(\begin{array}{ll}1 & 0 \\ 0 & 3\end{array}\right)$ and $\mathrm{B}=\left(\begin{array}{cc}2 & -1 \\ -1 & 2 / 3\end{array}\right)$.

Let $g(A)$ be the Kac - Moody Lie algebra associated with the GCM A . In the usual notation $\mathrm{g}(\mathrm{A})$ denote the Kac-Moody algebra associated with $G_{2}$. Let $\pi$ be the set of simple roots of $g(\mathrm{~A})$. Let $\tilde{\mathrm{A}}=\left(\left(\alpha_{i}, \alpha_{\mathrm{j}}\right), \mu_{\tilde{\mathrm{A}}}\left(\alpha_{i}, \alpha_{j}\right)\right)$ be the fuzzy set on $\pi \times \pi$ given by (1).

The following lemmas describe some properties of this fuzzy set:

Lemma 1: Support of the fuzzy set

$\widetilde{\mathrm{A}}=\left\{\left(\alpha_{1}, \alpha_{1}\right),\left(\alpha_{2}, \alpha_{2}\right),\left(\alpha_{1}, \alpha_{2}\right),\left(\alpha_{2}, \alpha_{1}\right)\right\}=X$.

Proof: By the definition of support,

$\operatorname{Supp}(\tilde{\mathrm{A}})=\left\{\left(\alpha_{\mathrm{i}}, \alpha_{\mathrm{j}}\right) \in \mathrm{X} / \mu\left(\alpha_{\mathrm{i}}, \alpha_{\mathrm{j}}\right)>0\right\}$

$=\left\{\left(\alpha_{1}, \alpha_{1}\right),\left(\alpha_{2}, \alpha_{2}\right),\left(\alpha_{1}, \alpha_{2}\right),\left(\alpha_{2}, \alpha_{1}\right)\right\}=X$.

Lemma 2: Height of the fuzzy set $\left\{\left(\alpha_{i}, \alpha_{j}\right), \mu_{\tilde{\mathrm{A}}}\left(\alpha_{i}, \alpha_{j}\right)\right\}$ is 1 .

Proof: Height of the fuzzy set is 1 because the maximum membership grade attained is 1 .

Lemma 3: Core of the fuzzy set $\tilde{\mathrm{A}}$ is $\left\{\left(\alpha_{1}, \alpha_{1}\right)\right\}$

Proof: By the definition of core,

$\operatorname{Core}(\tilde{A})=\left\{\left(\alpha_{i}, \alpha_{j}\right) \in X / \mu_{\tilde{A}}\left(\alpha_{i}, \alpha_{j}\right)=1\right\}=\left\{\left(\alpha_{1}, \alpha_{1}\right)\right\}$

Lemma 4: The fuzzy set $\widetilde{A}$ is normal.

Proof: Since $\operatorname{Sup}_{\left(\alpha_{i}, \alpha_{j}\right)} \mu_{\widetilde{A}}\left(\alpha_{i}, \alpha_{j}\right)=1$, the fuzzy set is normal.

Theorem 5: Let A be an indecomposable with the GCM $\left(\begin{array}{cc}2 & -1 \\ -3 & 2\end{array}\right)$. Let $\mathrm{g}(\mathrm{A})$ be the Kac - Moody algebra associated with $\mathrm{G}_{2}$. Let $\tilde{\mathrm{A}}$ be the fuzzy set defined on $\pi \times \pi$ given by (1). Then for $G_{2}$, the $\alpha$ - level sets and strong $\alpha$ - level sets for $\alpha=1,1 / 2,1 / 3, \ldots, 1 / \mathrm{k} \ldots$ are given by,
i. $A_{1}=\left\{\left(\alpha_{1}, \alpha_{1}\right)\right\}$
ii. $A_{1 / 2}=\left\{\left(\alpha_{1}, \alpha_{1}\right),\left(\alpha_{1}, \alpha_{2}\right),\left(\alpha_{2}, \alpha_{1}\right)\right\}$
iii. $A_{1 / 3}=\left\{\left(\alpha_{1}, \alpha_{1}\right),\left(\alpha_{2}, \alpha_{2}\right),\left(\alpha_{1}, \alpha_{2}\right),\left(\alpha_{2}, \alpha_{1}\right)\right\},\left|A_{1 / 3}\right|=4$
iv. $A_{1 / 4}=A_{1 / 3}, A_{1} \subset A_{1 / 2} \subset A_{1 / 3}=A_{1 / 4}=\ldots=A_{1 / k}=\ldots . X$,
$\left|\mathrm{A}_{1 / \mathrm{k}}\right|=4, \mathrm{k}=3,4, \ldots$
v. $A_{1 / 2}^{\prime}=\left\{\left(\alpha_{1}, \alpha_{1}\right)\right\}$.
vi. $A_{1 / 3}^{\prime}=\left\{\left(\alpha_{1}, \alpha_{1}\right),\left(\alpha_{1}, \alpha_{2}\right),\left(\alpha_{2}, \alpha_{1}\right)\right\}$. 
vii $\mathrm{A}_{1 / 4}^{\prime}=\left\{\left(\alpha_{1}, \alpha_{1}\right),\left(\alpha_{2}, \alpha_{2}\right),\left(\alpha_{1}, \alpha_{2}\right),\left(\alpha_{2}, \alpha_{1}\right)\right\}=\mathrm{X},\left|\mathrm{A}_{1 / 4}^{\prime}\right|=4$ viii. $A_{1 / 5}^{\prime}=A_{1 / 4}^{\prime}$,

$$
\begin{gathered}
\mathrm{A}_{1 / 2}^{\prime} \subset \mathrm{A}_{1 / 3}^{\prime} \subset \mathrm{A}_{1 / 4}^{\prime}=\mathrm{A}_{1 / 5}^{\prime}=\ldots=\mathrm{A}_{1 / \mathrm{k}}^{\prime}=\ldots=\mathrm{X}, \\
\left|A_{1 / k}^{\prime}\right|=4, k=4,5, \ldots
\end{gathered}
$$

Proof: (i) To compute 1- level Set:

$\mathrm{A}_{1}=\left\{\left(\alpha_{\mathrm{i}}, \alpha_{\mathrm{j}}\right) \in \mathrm{X} / \mu_{\tilde{\mathrm{A}}}\left(\alpha_{\mathrm{i}}, \alpha_{\mathrm{j}}\right) \geq 1\right\}=\left\{\left(\alpha_{1}, \alpha_{1}\right)\right\}$

(ii) To compute $1 / 2$-level Set:

$A_{1 / 2}=\left\{\left(\alpha_{i}, \alpha_{j}\right) \in X / \mu_{\tilde{\AA}}\left(\alpha_{i}, \alpha_{j}\right) \geq 1 / 2\right\}=\left\{\left(\alpha_{1}, \alpha_{1}\right),\left(\alpha_{1}, \alpha_{2}\right),\left(\alpha_{2}, \alpha_{1}\right)\right\}$

(iii) To compute $1 / 3$-level set:

$A_{1 / 3}=\left\{\left(\alpha_{i}, \alpha_{j}\right) \in X / \mu_{\tilde{A}}\left(\alpha_{i}, \alpha_{j}\right) \geq 1 / 3\right\}$

$$
=\left\{\left(\alpha_{1}, \alpha_{1}\right),\left(\alpha_{2}, \alpha_{2}\right),\left(\alpha_{1}, \alpha_{2}\right),\left(\alpha_{2}, \alpha_{1}\right)\right\}=X,\left|A_{1 / 3}\right|=4
$$

(iv) To compute $1 / 4$ - level set:

$\mathrm{A}_{1 / 4}=\left\{\left(\alpha_{\mathrm{i}}, \alpha_{\mathrm{j}}\right) \in \mathrm{X} / \mu_{\tilde{\mathrm{A}}}\left(\alpha_{\mathrm{i}}, \alpha_{\mathrm{j}}\right) \geq 1 / 4\right\}=\mathrm{A}_{1 / 3}$

From the above result,

$\mathrm{A}_{1} \subset \mathrm{A}_{1 / 2} \subset \mathrm{A}_{1 / 3}=\mathrm{A}_{1 / 4}=\ldots=\mathrm{A}_{1 / \mathrm{k}}=\ldots \mathrm{X}$,

$\left|\mathrm{A}_{1 / \mathrm{k}}\right|=4$, for $\mathrm{k}=3,4, \ldots$

(v) To compute strong $1 / 2$ - level set:

$\mathrm{A}_{1 / 2}^{\prime}=\left\{\left(\alpha_{\mathrm{i}}, \alpha_{\mathrm{j}}\right) \in \mathrm{X} / \mu_{\tilde{\mathrm{A}}}\left(\alpha_{\mathrm{i}}, \alpha_{\mathrm{j}}\right)>1 / 2\right\}=\left\{\left(\alpha_{1}, \alpha_{1}\right)\right\}$

(vi) To compute strong $1 / 3$-level set:

$\mathrm{A}_{1 / 3}^{\prime}=\left\{\left(\alpha_{\mathrm{i}}, \alpha_{\mathrm{j}}\right) \in \mathrm{X} / \mu_{\tilde{\mathrm{A}}}\left(\alpha_{\mathrm{i}}, \alpha_{\mathrm{j}}\right)>1 / 3\right\}$

$=\left\{\left(\alpha_{1}, \alpha_{1}\right),\left(\alpha_{1}, \alpha_{2}\right),\left(\alpha_{2}, \alpha_{1}\right)\right\}$

(vii) To compute strong $1 / 4$ - level set:

$\mathrm{A}_{1 / 4}^{\prime}=\left\{\left(\alpha_{\mathrm{i}}, \alpha_{\mathrm{j}}\right) \in \mathrm{X} / \mu_{\tilde{\mathrm{A}}}\left(\alpha_{\mathrm{i}}, \alpha_{\mathrm{j}}\right)>1 / 4\right\}$

$=\left\{\left(\alpha_{1}, \alpha_{1}\right),\left(\alpha_{2}, \alpha_{2}\right),\left(\alpha_{1}, \alpha_{2}\right),\left(\alpha_{2}, \alpha_{1}\right)\right\}=X$,

$\left|\mathrm{A}_{1 / 4}^{\prime}\right|=4$.

(viii) To compute strong $1 / 5$ - level set:

$\mathrm{A}_{1 / 5}^{\prime}=\left\{\left(\alpha_{\mathrm{i}}, \alpha_{\mathrm{j}}\right) \in \mathrm{X} / \mu_{\tilde{\mathrm{A}}}\left(\alpha_{\mathrm{i}}, \alpha_{\mathrm{j}}\right)>1 / 5\right\}=\mathrm{A}_{1 / 4}^{\prime}$

From the above results,

$\mathrm{A}_{1 / 2}^{\prime} \subset \mathrm{A}_{1 / 3}^{\prime} \subset \mathrm{A}_{1 / 4}^{\prime}=\mathrm{A}_{1 / 5}^{\prime} .=\ldots=\mathrm{A}_{1 / \mathrm{k}}^{\prime}=\ldots=\mathrm{X}$.

Since $A_{1 / 3}^{\prime}=A_{1 / k}^{\prime},\left|A_{1 / k}^{\prime}\right|=4$ for $k=4,5, \ldots$

Lemma 6: A fuzzy set $\tilde{A}$ corresponding to the finite type of Kac-Moody algebra $\mathrm{G}_{2}$, defined by (1) is convex.

Proof: Consider the finite type of family $\mathrm{G}_{2}$. The table showing all possible membership grades for the elements of $\mathrm{X}$ and the conditions for checking convexity is listed below:

Table 1. Possible membership grades attained by the elements of $X$

\begin{tabular}{|c|c|c|c|}
\hline$\mu_{\tilde{\mathrm{A}}}\left(\mathrm{x}_{1}\right)$ & $\mu_{\tilde{\mathrm{A}}}\left(\mathrm{x}_{2}\right)$ & $\mu_{\tilde{\mathrm{A}}}\left[\lambda \mathrm{x}_{1}+(1-\lambda) \mathrm{x}_{2}\right]$ & $\min \left[\left(\mu_{\tilde{\mathrm{A}}}\left(\mathrm{x}_{1}\right), \mu_{\tilde{\mathrm{A}}}\left(\mathrm{x}_{2}\right)\right]\right.$ \\
\hline 1 & 1 & 1 & 1 \\
\hline $1 / 2$ & 1 & $(2-\lambda) / 2$ & $1 / 2$ \\
\hline 1 & $1 / 2$ & $(1+\lambda) / 2$ & $1 / 2$ \\
\hline $1 / 2$ & $1 / 2$ & $1 / 2$ & $1 / 2$ \\
\hline $1 / 3$ & $1 / 3$ & $1 / 3$ & $1 / 3$ \\
\hline $1 / 3$ & 1 & $(3-2 \lambda) / 3$ & $1 / 3$ \\
\hline 1 & $1 / 3$ & $(2 \lambda+1) / 3$ & $1 / 3$ \\
\hline $1 / 3$ & $1 / 2$ & $(3-\lambda) / 6$ & $1 / 3$ \\
\hline $1 / 2$ & $1 / 3$ & $(\lambda+2) / 6$ & $1 / 3$ \\
\hline
\end{tabular}

From the above table, we see that for every $\mathrm{x}_{1}, \mathrm{x}_{2} \in \mathrm{X}$ and $\forall \lambda \in[0,1], \mu_{\tilde{A}}\left[\lambda x_{1}+(1-\lambda) x_{2}\right] \geq \min \left[\mu_{\tilde{A}}\left(x_{1}\right), \mu_{\tilde{A}}\left(x_{2}\right)\right]$.
Hence the fuzzy set $\widetilde{A}$ corresponding to $G_{2}$ of finite type of Kac-Moody algebra is convex.

Theorem 7: Let $\widetilde{\mathrm{A}}$ be the fuzzy set defined on $\pi \times \pi$, where $\pi$ denotes the root basis for the finite Kac-Moody algebra given by equation (1). Then the $\alpha$-cut decomposition for the fuzzy set $\tilde{A}$ for the finite family $G_{2}$ is $1 A_{1} \cup 1 / 2 A_{1 / 2} \cup$ $1 / 3 \mathrm{~A}_{1 / 3}$.

Proof: From the Theorem No [5] for the finite family $\mathrm{G}_{2}$, We have $A_{1} \subset A_{1 / 2} \subset A_{1 / 3}=A_{1 / 4}=\ldots=A_{1 / k}=\ldots=X . \quad B y$ the definition of $\alpha$-cut decomposition,

The $\alpha$-cut decomposition for the fuzzy set $\tilde{\mathrm{A}}$ for the finite family $\mathrm{G}_{2}: 1 \mathrm{~A}_{1} \cup 1 / 2 \mathrm{~A}_{1 / 2} \cup 1 / 3 \mathrm{~A}_{1 / 3}$.

Lemma 8: Let $A$ be an indecomposable with the GCM $\left(\begin{array}{cc}2 & -1 \\ -3 & 2\end{array}\right)$. Let $\mathrm{g}(\mathrm{A})$ be the Kac - Moody Lie algebra associated with $\mathrm{G}_{2}$. Let $\tilde{\mathrm{A}}$ be the fuzzy set defined on $\pi \times \pi$ given by equation (1). Then $\tilde{\mathrm{A}}$ has the following properties:

(a) The cardinality $|\tilde{\mathrm{A}}|=7 / 3$.

(b) Relative cardinality $\|\tilde{\mathrm{A}}\|=7 / 12$.

(c) The membership function of the complement of a normalized fuzzy set $\tilde{\mathrm{A}}$ corresponding to the finite type $\mathrm{G}_{2}, \forall\left(\alpha_{\mathrm{i}}, \alpha_{\mathrm{j}}\right) \in \mathrm{X}$ are listed below :

$$
\begin{aligned}
& \text { i. } \mu_{\mathbb{C} \tilde{\mathrm{A}}}\left(\alpha_{\mathrm{i}}, \alpha_{\mathrm{j}}\right)=0 \mathrm{i}=\mathrm{j}=1 . \\
& \text { ii. } \mu_{\widetilde{C} \tilde{\mathrm{A}}}\left(\alpha_{\mathrm{i}}, \alpha_{\mathrm{i}+1}\right)=1 / 2, \mathrm{i}=1 . \\
& \text { iii. } \mu_{\widetilde{C} \tilde{\mathrm{A}}}\left(\alpha_{\mathrm{i}}, \alpha_{\mathrm{j}}\right)=2 / 3 \text { for } \mathrm{i}=\mathrm{j}=2 . \\
& \text { iv. } \mathrm{FC}(\tilde{\mathrm{A}})=1+\frac{1}{2} \cdot \frac{1}{3}+\frac{1}{3} \cdot \frac{1}{4}
\end{aligned}
$$

Proof: From equation (1), the fuzzy set $\tilde{\mathrm{A}}$ corresponding to the finite type of Kac-Moody algebra associated with $\mathrm{G}_{2}$ contains 1 element in $\mathrm{X}$ having membership grade 1,2 elements in $\mathrm{X}$ having membership grade $1 / 2$ and 1 element in $\mathrm{X}$ having membership grade $1 / 3$.

By the definition of cardinality,

(a) $|\tilde{\mathrm{A}}|=\sum_{\mathrm{x} \in \mathrm{X}} \mu_{\tilde{\mathrm{A}}}(\mathrm{x})=7 / 3$.

(b) $\|\tilde{\mathrm{A}}\|=\frac{|\tilde{\mathrm{A}}|}{|\mathrm{X}|}=\frac{7}{12}$

(c) Since the fuzzy set $\tilde{A}$ corresponding to the classical algebra $\mathrm{G}_{2}$, is normal, $\forall\left(\alpha_{\mathrm{i}}, \alpha_{\mathrm{j}}\right) \in \mathrm{X}$, the membership function of the complement of a normalized fuzzy set $\tilde{\mathrm{A}}$ are listed below :

i. $\mu_{C \tilde{\mathrm{A}}}\left(\alpha_{\mathrm{i}}, \alpha_{\mathrm{j}}\right)=1-1=0$ for $\mathrm{i}=\mathrm{j}=1$

ii. For, $i=1, \mu_{\mathbb{C} \tilde{\mathrm{A}}}\left(\alpha_{\mathrm{i}}, \alpha_{\mathrm{i}}\right)=1-1 / 2=1 / 2$

iii. For $\mathrm{i}=\mathrm{j}=2, \mu_{\subset \widetilde{A}}\left(\alpha_{i}, \alpha_{j}\right)=1-(1 / 3)=2 / 3$.

iv. By the definition of

$$
\begin{gathered}
\mathrm{FC}(\tilde{\mathrm{A}})=\sum \frac{\alpha}{n_{\alpha}} \text { where } \mathrm{n} \alpha=\operatorname{SC}(\mathrm{A} \alpha), \\
=1+\frac{1}{2} \cdot \frac{1}{3}+\frac{1}{3} \cdot \frac{1}{4}
\end{gathered}
$$

Lemma 9: Let $A$ be an indecomposable with the GCM $\left(\begin{array}{cc}2 & -1 \\ -3 & 2\end{array}\right)$. Let $\mathrm{g}(\mathrm{A})$ be the $\mathrm{Kac}-$ Moody Lie algebra 
associated with $\mathrm{G}_{2}$. Let $\tilde{\mathrm{A}}$ be the fuzzy set defined on $\pi \times \pi$ given by equation (1). Then the Dilation and Concentration are given by

(i) $\operatorname{Dil}(\tilde{\mathrm{A}})=\{1, .7, .5\}$

(ii) $\operatorname{Con}(\tilde{\mathrm{A}})=\{1, .25, .09\}$.

Proof: (i) By the definition of Dilation,

$\operatorname{Dil}(\tilde{\mathrm{A}})=[\mathrm{A}(\mathrm{x})]^{0.5}$ for all $\mathrm{x}$ in $\widetilde{\mathbf{A}}$,

$$
=\{1, .7, .5\}
$$

(ii) By the definition of Concentration,

$$
\begin{aligned}
\operatorname{con}(\tilde{\mathrm{A}})(\mathrm{x}) & =[\tilde{\mathrm{A}}]^{2} \forall \mathrm{x} \in \tilde{\mathrm{A}} \\
& =\{1, .25, .09\} .
\end{aligned}
$$

Lemma 10: Let $A$ be an indecomposable with the GCM $\left(\begin{array}{cc}2 & -1 \\ -3 & 2\end{array}\right)$. Let $g(A)$ be the Kac - Moody Lie algebra associated with $\mathrm{G}_{2}$. Let $\tilde{\mathrm{A}}$ be the fuzzy set defined on $\pi \times \pi$ given by equation (1). Then the contrast intensification of a fuzzy set $\tilde{\mathrm{A}}$ is given by

$$
\text { Int } \tilde{\mathrm{A}}(\mathrm{x})=\left\{\begin{array}{l}
.5, .18, \text { if } 0 \leq \tilde{\mathrm{A}}(\mathrm{x}) \leq .5 \\
1, \text { if } .5 \leq \tilde{\mathrm{A}}(\mathrm{x}) \leq 1
\end{array}\right\}
$$

Proof: By the definition of contrast intensification of a fuzzy set $\tilde{\mathrm{A}}$,

$$
\begin{aligned}
\operatorname{Int}(\tilde{\mathrm{A}})(\mathrm{x}) & =2[\tilde{\mathrm{A}}(\mathrm{x})]^{2} \text { for } 0 \leq \tilde{\mathrm{A}}(\mathrm{x}) \leq 0.5 \\
& =2(.5)^{2} \text { and } 2(.3)^{2} \\
& =\{.5, .18\}
\end{aligned}
$$

$\operatorname{Int}(\tilde{\mathrm{A}})(\mathrm{x})=1-2[1-\tilde{\mathrm{A}}(\mathrm{x})]^{2}$ for $0.5 \leq \mathrm{A}(\mathrm{x}) \leq 1$

$$
=1-2[1-1]^{2}=1 \text {. }
$$

Hence,

$$
\text { Int } \tilde{\mathrm{A}}(\mathrm{x})=\left\{\begin{array}{l}
.5, .18, \text { if } \quad 0 \leq \tilde{\mathrm{A}}(\mathrm{x}) \leq .5 \\
1, \text { if } .5 \leq \tilde{\mathrm{A}}(\mathrm{x}) \leq 1
\end{array}\right\}
$$

Lemma 11: Let $A$ be an indecomposable with the GCM $\left(\begin{array}{cc}2 & -1 \\ -3 & 2\end{array}\right)$. Let $\mathrm{g}(\mathrm{A})$ be the $\mathrm{Kac}$ - Moody Lie algebra associated with $\mathrm{G}_{2}$. Let $\tilde{\mathrm{A}}$ be the fuzzy set defined on $\pi \times \pi$ given by equation (1). Then the Hamming distance and Euclidean distances are given by

$$
\operatorname{Hamm}(\tilde{\mathrm{A}})=2 / 3 \& \operatorname{Euc}(\tilde{\mathrm{A}})=(10 / 9)^{1 / 2}
$$

Proof: By the definition of Hamming distance and Euclidean distance,

$$
\begin{aligned}
& \operatorname{Hamm}(\tilde{\mathrm{A}})=\sum\left|\tilde{\mathrm{A}}(\mathrm{x})-\tilde{\mathrm{A}}^{\prime}(\mathrm{x})\right| \\
& =(1-0)+\left(\frac{1}{2}-\frac{1}{2}\right)+\left(\frac{1}{2}-\frac{1}{2}\right)+\left(\frac{1}{3}-\frac{2}{3}\right)=\frac{2}{3} \\
& \operatorname{Euc}(\tilde{\mathrm{A}})=\left[\sum\left(\tilde{\mathrm{A}}(\mathrm{x})-\tilde{\mathrm{A}}^{\prime}(\mathrm{x})\right)^{2}\right]^{1 / 2} \\
& =\left[(1)^{2}+\left(\frac{-1}{3}\right)^{2}\right]^{1 / 2}=\left(\frac{10}{9}\right)^{1 / 2} .
\end{aligned}
$$

Lemma 12: Let $A$ be an indecomposable with the GCM $\left(\begin{array}{cc}2 & -1 \\ -3 & 2\end{array}\right)$. Let $g(\mathrm{~A})$ be the $\mathrm{Kac}-$ Moody Lie algebra associated with $\mathrm{G}_{2}$. Let $\tilde{\mathrm{A}}$ be the fuzzy set defined on $\pi \times \pi$ given by equation (1).The index is given by the following functions which are based on the $\alpha$-cut with $\alpha=0.5$ :
(i) $f_{H}(\tilde{A})=\frac{1}{3}$
(ii) $f_{E}(\tilde{A})=\frac{1}{9}$

The normalized values of the index functions are given by (iii) $f_{H}{ }^{*}(\tilde{A})=\frac{1}{6} \quad$ (iv) $f_{E}{ }^{*}(\tilde{A})=\frac{1}{9}$

Proof: By the definition,

(i) $\mathrm{f}_{\mathrm{H}}(\tilde{\mathrm{A}})=\sum\left|\tilde{\mathrm{A}}(\mathrm{x})-\tilde{\mathrm{A}}_{0.5}(\mathrm{x})\right|=\frac{1}{3}$

(ii) $\mathrm{f}_{\mathrm{E}}(\tilde{\mathrm{A}})=\left[\sum\left(\tilde{\mathrm{A}}(\mathrm{x})-\tilde{\mathrm{A}}_{0.5}(\mathrm{x})\right)^{2}\right]^{1 / 2}=\frac{1}{9}$

By the definition of the normalized values of the index functions are given by
(iii) $\mathrm{f}_{\mathrm{H}}{ }^{*}(\tilde{\mathrm{A}})=\frac{\left(2 \mathrm{f}_{\mathrm{H}}(\widetilde{\mathrm{A}})\right)}{\#(\operatorname{supp}(\tilde{\mathrm{A}}))}=\frac{1}{6}$
(iv) $\mathrm{f}_{\mathrm{E}}{ }^{*}(\tilde{\mathrm{A}})=\frac{\left(2 \mathrm{f}_{\mathrm{E}}(\tilde{\mathrm{A}})\right)}{[\#(\operatorname{supp}(\tilde{\mathrm{A}}))]^{0.5}}=\frac{1}{9}$

\section{CONCLUSION}

We can further compute the level cuts for various families of affine, hyperbolic and non hyperbolic of Kac-Moody algebras; Other structural properties on the fuzzy nature of these algebras can also be studied.

\section{REFERENCES}

[1] Ganesh.M., 2009. Introduction to fuzzy sets and fuzzy logic. New Delhi: PHI Learning Private Limited.

[2] Kac.V.G., 1990. Infinite Dimensional Lie Algebra, 3rd ed., Cambridge : Cambridge University Press.

[3] Moody,R.V., 1968 . A new class of Lie algebras. J. Algebra. 10, pp. 211-230.

[4] Uma Maheswari, A, \& Krishnaveni, S, 2012, "Fuzziness on the Root System of Finite Type of Kac-Moody Algebras", Proceedings of the International Conference on Mathematical Modeling And Applied Soft Computing, Vol 1 ISBN 978-81-92375

[5] Uma Maheswari, A., 2012, "Fuzzy Decomposition on the affine Type of Kac-Moody Algebras ", IJPAM Vol 80. No $12012-75$ - 91.

[6] Uma Maheswari, A., 2012, "Fuzzy Decomposition on the affine Type of Kac-Moody Algebras $\mathrm{F}_{4}{ }^{(1)}, \mathrm{E}_{7}{ }^{(1)} \mathrm{E}_{8}{ }^{(1)}$ $\& \mathrm{G}_{2}{ }^{(1)}$ ", IJSER Vol 3-Issue 7.

[7] Uma Maheswari, A., \& Gayathri, V, 2012, "Fuzzy Properties On Finite type Of Kac-Moody algebras “, Lecture notes on Springer-Verlag CCIS 305 pp-352-363.

[8] Uma Maheswari, A., 2012, "Fuzziness on the hyperbolic algebras $\mathrm{HG}_{2}$ ", Mathematical Sciences International Research Journals- ISSN- 2278- 8697.

[9] Uma Maheswari, A., \& Krishnaveni, S., 2012, "Fuzziness on the Root System of Finite Type of KacMoody Algebras $\mathrm{E}_{6}, \mathrm{E}_{7} \& \mathrm{E}_{8}$ ", IEEE Proceedings of the International Workshop on Mathematical Modeling ICECCS 2012, Kochin.

[10] Wan Zhe-Xian.,1991. Introduction to Kac-Moody Algebra. Singapore : World Scientific Publishing Co.Pvt.Ltd.

[11] Zimmermann. H.J, 2001. Fuzzy Set Theory and its applications, 4th ed., London: Kluwer Academic Publisher. 